Check for updates

Cite this: Phys. Chem. Chem. Phys., 2021, 23, 14352

Received 11th April 2021,

Accepted 16th June 2021

DOI: $10.1039 / \mathrm{d} 1 \mathrm{cp} 01584 \mathrm{~b}$

rsc.li/pccp

\section{Sign flipping of spontaneous polarization in vapour-deposited films of small polar organic molecules $\dagger$}

\author{
Georgios M. Tourlakis, Sotirios Alexandros T. Adamopoulos, Irini K. Gavra, \\ Alexandros A. Milpanis, Liveria F. Tsagri, Aikaterini Sofia G. Pachygianni, \\ Stylianos S. Chatzikokolis and Athanassios A. Tsekouras (D) *
}

\begin{abstract}
Films of polar molecules vapour-deposited on sufficiently cold substrates are not only amorphous, but also exhibit charge polarization across their thickness. This is an effect known for 50 years, but it is very poorly understood and no mechanism exists in the literature that can explain and predict it. We investigated this bulk effect for 18 small organic molecules as a function of substrate temperature $(30-130 \mathrm{~K})$. We found that, as a rule, alcohol films have the negative end on the vacuum side at all temperatures. Alkyl acetates and toluene showed positive voltages which reached a maximum around the middle of the temperature range investigated. Tetrahydrofuran showed positive voltages which dropped with increasing deposition temperature. Diethyl ether, acetone, propanal, and butanal showed positive film voltages at low temperatures, negative at intermediate temperatures and again positive voltages at higher temperatures. In all cases, film voltages were monitored during heating leading to film evaporation. Film voltages were irreversibly eliminated before film elimination, but voltage profiles during temperature ramps differed vastly depending on compound and deposition temperature. In general, there was a gradual voltage reduction, but propanal, butanal, and diethyl ether showed a change in voltage sign during temperature ramp in films deposited at low temperatures. All these data expand substantially the experimental information regarding spontaneous polarization in vapour-deposited films, but still require complementary measurements as well as numerical simulations for a detailed explanation of the phenomenon.
\end{abstract}

\section{Introduction}

Molecules from the gas phase condense into a solid when they come in contact with a sufficiently cold substrate. If the molecules are polar, i.e., they have an electric dipole moment, and the substrate temperature is below a certain threshold, the condensate exhibits an electric potential difference between the side in contact with the substrate and that on the outside. This is rather unexpected. It is well known that when molecules are cooled, they form condensed phases, first liquid, later solid. As their temperature is reduced, they are always in contact with each other. In the absence of external fields, space is isotropic and no long-range electric fields or voltages are established; solids, either crystalline or amorphous do not form with molecules aligned along any axis. In contrast, molecules forming solids with abrupt cooling and directly from the gas phase

Physical Chemistry Laboratory, Department of Chemistry, National and Kapodistrian University of Athens, Panepistimiopolis, Zografos, GR-15784, Greece. E-mail: thanost@chem.uoa.gr

$\dagger$ Electronic supplementary information (ESI) available. See DOI: 10.1039/d1cp01584b partially align themselves along the surface normal and give rise to sizeable electric potential differences.

This counter-intuitive phenomenon was reported 50 years ago, ${ }^{1}$ but has not been studied extensively either at cryogenic temperatures $^{2-31}$ or near room temperature $;^{32-47}$ the small number of research groups involved in such studies tended to concentrate on very few compounds, when a lot of information is lacking. What is known about this effect is limited to four facts: (i) condensing molecules need to have a dipole moment; (ii) voltages observed are proportional to the thickness of the condensate films, i.e., this is a bulk rather than a surface effect; (iii) the nature of the substrate is immaterial; (iv) the films thus created exist in a metastable state and the voltage is eliminated when the films are heated and not recovered upon re-cooling. ${ }^{23}$ The significance of other factors has not been established. Some of these factors are expected to be the magnitude of the electric dipole moment, molecular polarizability, substrate temperature and its value relative to characteristic temperatures of the condensing molecules (such as melting point, glass transition temperature, critical temperature), rate of condensation, presence of other molecules (or absence thereof, i.e., vacuum), 
collision energy of the impinging molecules. There may be other factors as well which are not easily identified or controlled and have not been studied in any significant detail. The sign and magnitude of the observed voltage depends primarily on the identity of the condensing molecules and on the substrate temperature. Some molecules have been reported to produce negative voltages (of the outer film surface with respect to the substrate side), others positive; a few are known to change voltage sign depending on substrate temperature. ${ }^{1}$

The metastable state of the films formed and electric field alignment along the surface normal indicates that the films are formed layer by layer; molecules are trapped to some extent in configurations that are not the most stable ones when they are surrounded by other molecules from all sides, yet they are in local energy minima, while they are still on the surface. Such considerations have been used to explain other properties of vapour-deposited films. ${ }^{48,49}$ Experiments $^{50-71}$ have looked, among other things, at extended temperature range with persistently low heat capacities, at delayed appearance of glass transition temperatures, and at density effects. They find the most interesting deposition temperature range just below the glass transition temperature of some compounds, whereas spontaneous polarization is more pronounced at lower temperatures in general. A few simulations have indicated that layering of impinging particles and a state of surface molecules similar to pre-melting can explain thermodynamic stability and charge polarization. ${ }^{61,72}$ Unfortunately, these simulations have not yet gone far enough to provide predictive capability for given molecular structures or show voltage dependence on deposition temperature.

Our aim was to provide experimental data while trying to understand the phenomenon of spontaneous polarization. We have studied a variety of compounds belonging to different homologous series in an attempt to get a big picture and establish trends. We present here several sets of data for 18 molecules as a function of substrate temperature. We did see some trends and found surprising variability in behaviour for various substances. For each set we have collected additional information regarding the evolution of the film voltage as its temperature is raised leading to the eventual elimination of the voltage and then the evaporation of the film itself. We conclude with a brief discussion of the emerging picture for the spontaneous polarization of cryo-deposited films.

\section{Experimental}

The experiments were conducted in a triple cross, high vacuum chamber $^{23}$ equipped with a Kelvin probe (for measuring film voltages), a tube doser (for directing known amounts of gas onto the substrate), a helium closed-cycle refrigerator cooling a polycrystalline platinum foil, and a quadrupole mass spectrometer for monitoring the gas phase composition. A notable addition to the system involves a sparse screen made of 11 parallel, equally-spaced lengths of nickel-plated copper wire mounted on a $12 \mathrm{~cm}$ diameter (used) copper gasket, positioned between the triple cross and the mass spectrometer, and held at $-230 \mathrm{~V}$ to prevent electrons ejected by the spectrometer from reaching the sample or the Kelvin probe. This addition causes an imperceptible reduction in the pumping speed, but allows the unbiased measurement of positive film voltages. Not all data were recorded with this configuration, hence in some cases the mass spectrometer was kept off and chamber pressure was used as its proxy to monitor desorption.

Known amounts of a given gas at room temperature were delivered directly on the cold substrate, with a miniscule fraction escaping condensation. The number of molecules deposited was calculated based on the reduction of pressure in the gas reservoir of known volume and temperature. ${ }^{23}$ Deposition time was of the order of 60 seconds, while the total number of molecules was of the order of $10^{18} \mathrm{~cm}^{-2}$ or, equivalently, $10^{3}$ monolayers (ML). Within a couple of minutes after deposition, the sample was rotated in front of the Kelvin probe and we measured the film voltage and started a temperature ramp at a rate of $0.25 \mathrm{~K} \mathrm{~s}^{-1}$. Chamber pressure varied from $0.2 \times 10^{-8}$ Torr while on standby to $0.05 \times 10^{-8}$ Torr during cooling, with short bursts up to $5 \times 10^{-7}$ Torr during film deposition and peaking around $3 \times 10^{-6}$ torr upon film desorption.

\section{Results}

Data were recorded for a variety of chemical compounds, listed in Table 1. During the temperature ramp after the film deposition, the voltage (positive or negative) gradually dropped to zero; in many cases it would first increase in absolute value and then gradually decrease; in yet other cases it would quickly drop so far as to switch sign before becoming eliminated. Some compounds showed variation of the magnitude of the film voltage as a function of substrate temperature, but maintained constant voltage sign for all temperatures studied; others, though, showed one or two changes of initial voltage sign over the temperature range studied. The temperature range was determined on the low side by our apparatus, i.e., around $30 \mathrm{~K}$, whereas on the high side it depended on the compound itself, namely, its desorption temperature and its ability to show non-zero voltage.

For each compound we present at least 3 figures. First, using the results for a single representative run we show film voltage variation as a function of film temperature along with the mass spectrometer signal indicating film desorption; arguably the phenomenon is described more accurately as sublimation and evaporation. On some occasions, when the mass spectrometer had been switched off, we display the chamber pressure as an adequate proxy for the mass spectrometer signal. These figures can be found in the ESI $\dagger$ as Fig. SA1-SA18. Then, for each compound, we show representative curves of film voltage scaled by the surface number density of deposited molecules as a function of film temperature which is ramped at a constant rate. For each curve the deposition temperature is the lowest temperature displayed. The third figure for each compound summarizes the results by displaying the (initial) film voltage at the deposition temperature as a function of that temperature, 
Table 1 Names, chemical formulae, dipole moments (in debye), indeces of refraction, calculated dielectric constants at infinite frequency and glass transition temperatures (in kelvin) of molecules used in this study

\begin{tabular}{|c|c|c|c|c|c|}
\hline Compound (abbreviation) & Formula & $\mu^{a}(\mathrm{D})$ & $n_{\mathrm{D}}{ }^{a}$ & $\varepsilon_{\infty}=\sqrt{n_{\mathrm{D}}}$ & $T_{\mathrm{g}}(\mathrm{K})$ \\
\hline 2-Propanol (2-PrOH) & $\mathrm{CH}_{3} \mathrm{CH}(\mathrm{OH}) \mathrm{CH}_{3}$ & 1.58 & 1.3776 & 1.17 & $121.3^{c d}$ \\
\hline tert-Butanol $(t-\mathrm{BuOH})$ & $\mathrm{C}\left(\mathrm{CH}_{3}\right)_{3} \mathrm{OH}$ & 1.66 & 1.3878 & 1.18 & $180^{d}$ \\
\hline 2-Pentanol (2-PeOH) & $\mathrm{CH}_{3}\left(\mathrm{CH}_{2}\right)_{2} \mathrm{CH}(\mathrm{OH}) \mathrm{CH}_{3}$ & 1.66 & 1.4053 & 1.19 & $140^{d}$ \\
\hline Ethylene glycol & $\left(\mathrm{CH}_{2} \mathrm{OH}\right)_{2}$ & 2.36 & 1.4318 & 1.20 & $150^{e}$ \\
\hline Methyl acetate (MeAc) & $\mathrm{CH}_{3} \mathrm{COOCH}_{3}$ & 1.72 & 1.3614 & 1.17 & \\
\hline Ethyl acetate (EtAc) & $\mathrm{CH}_{3} \mathrm{COOCH}_{2} \mathrm{CH}_{3}$ & 1.72 & 1.3723 & 1.17 & $118^{b}$ \\
\hline Propyl acetate (PrAc) & $\mathrm{CH}_{3} \mathrm{COO}\left(\mathrm{CH}_{2}\right)_{2} \mathrm{CH}_{3}$ & 1.72 & 1.3842 & 1.18 & \\
\hline Butyl acetate (BuAc) & $\mathrm{CH}_{3} \mathrm{COO}\left(\mathrm{CH}_{2}\right)_{3} \mathrm{CH}_{3}$ & 1.87 & 1.3941 & 1.18 & \\
\hline Pentyl acetate (PeAc) & $\mathrm{CH}_{3} \mathrm{COO}\left(\mathrm{CH}_{2}\right)_{4} \mathrm{CH}_{3}$ & 1.75 & 1.4023 & 1.18 & \\
\hline Acetone & $\mathrm{CH}_{3} \mathrm{COCH}_{3}$ & 2.88 & 1.3588 & 1.17 & $173^{b}$ \\
\hline Propanal & $\mathrm{CH}_{3} \mathrm{CH}_{2} \mathrm{CHO}$ & 2.52 & 1.3636 & 1.17 & \\
\hline
\end{tabular}

along with an extremum (most often a minimum), if one appeared at an intermediate temperature. In the latter cases, we also plot the temperature where the extremum appears versus the deposition temperature. In other cases, where the voltage drops gradually and monotonically, we show the temperature at which the voltage is halved and the rate of change of the voltage at that temperature again as a function of deposition temperature.

For a rudimentary analysis of the data we used the degree of dipole polarization (or orientation), $g$, as derived in ref. 23 .

$$
g=\frac{V A}{N} \frac{\varepsilon_{0} \varepsilon_{\infty}}{\mu}
$$

where $V$ is the film voltage; $N$ is the total number of molecules deposited; $A$ is the area on which they were deposited; $\frac{N}{A}$ is their surface number density; $\frac{V A}{N}$ is the film voltage scaled with the surface number density; $\varepsilon_{\infty}$ is the relative dielectric constant at infinite frequency (used for very low temperature dielectric response); $\mu$ is the dipole moment of the molecule; and $\frac{N}{A} \frac{\mu}{\varepsilon_{0} \varepsilon_{\infty}}$ is the expected voltage if all deposited molecules were positioned with their dipole moment vectors at right angles to the surface.

For a given compound any scaled voltage can be interpreted as degree of polarization using eqn (1). This has been done on some of the figures that display initial scaled voltage, by using a right axis for the degree of polarization. Predictions based on the index of refraction using the expression $\varepsilon_{\infty}=\sqrt{n_{\mathrm{D}}}$ were all around 1.2 (see Table 1), whereas other estimates ${ }^{73}$ placed these values close to 1.8. Hence, an average value of 1.5 was used for $\varepsilon_{\infty}$ for all compounds.

We present the results for different substances in order of increasing complexity in the dependence of film voltage on deposition temperature and in the form of the voltage profile during the subsequent temperature ramp.
Tetrahydrofuran (THF) was deposited at substrate temperatures ranging between $30.4 \mathrm{~K}$ and $72 \mathrm{~K}$. A typical example of a single run is shown in Fig. SA1 (ESI $\dagger$ ). The vacuum side of the film has a positive voltage with respect to its other side in contact with the substrate. For deposition of $5.4 \times 10^{2} \mathrm{ML}$ (using the customary definition of $1 \mathrm{ML}=10^{-8}$ molecules per $\mathrm{cm}^{2}$ ) or $0.89 \mu \mathrm{mol} \mathrm{cm} \mathrm{cm}^{-2}$ at $32.9 \mathrm{~K}$ the film voltage is $26 \mathrm{~V}$ which corresponds to $4.6 \times 10^{-17} \mathrm{~V} \mathrm{~cm}^{2}$ per molecule. As soon as the temperature ramp is started, the film voltage gradually drops to nearly 0 . THF is removed from the platinum foil above $150 \mathrm{~K}$, as seen from the chamber pressure. Desorption is partially hindered and delayed by the Kelvin probe tip sitting a fraction of a millimetre away from the sample causing the desorption peak to appear split in two components. This is seen for all compounds irrespective of whether the process is monitored via the mass spectrometer tuned to a fragment of the desorbing molecules or via the chamber pressure gauge.

Several runs presented in Fig. 1a for different deposition temperatures show positive voltages and closely overlapping profiles during the temperature ramps. Fig. 1b, which summarizes the initial scaled voltages and calculated degree of polarization, $g$, indicates that no film voltage is observed at deposition temperatures above $72 \mathrm{~K}$, yet the desorption temperature appears higher than $140 \mathrm{~K}$.

Five secondary and branched aliphatic monohydroxy alcohols were studied extending the study of normal primary alcohols. ${ }^{29}$ Results were similar in both sets. Fig. SA2-SA6 (ESI $\dagger$ ) depict representative single runs for 2-propanol, 2-butanol, 2-methyl 1-propanol (iso-butanol), 2-methyl 2-propanol (tert-butanol), and 2-pentanol. All alcohols exhibit negative film voltages upon deposition (see Fig. 2). These voltages become briefly more negative as the films are heated and then gradually go to zero. All these voltage profiles have similar shapes. For the most part starting values decrease in absolute value as the deposition temperature increases, but at the very low temperatures the voltage magnitude is not the highest, as seen in Fig. 2f. 

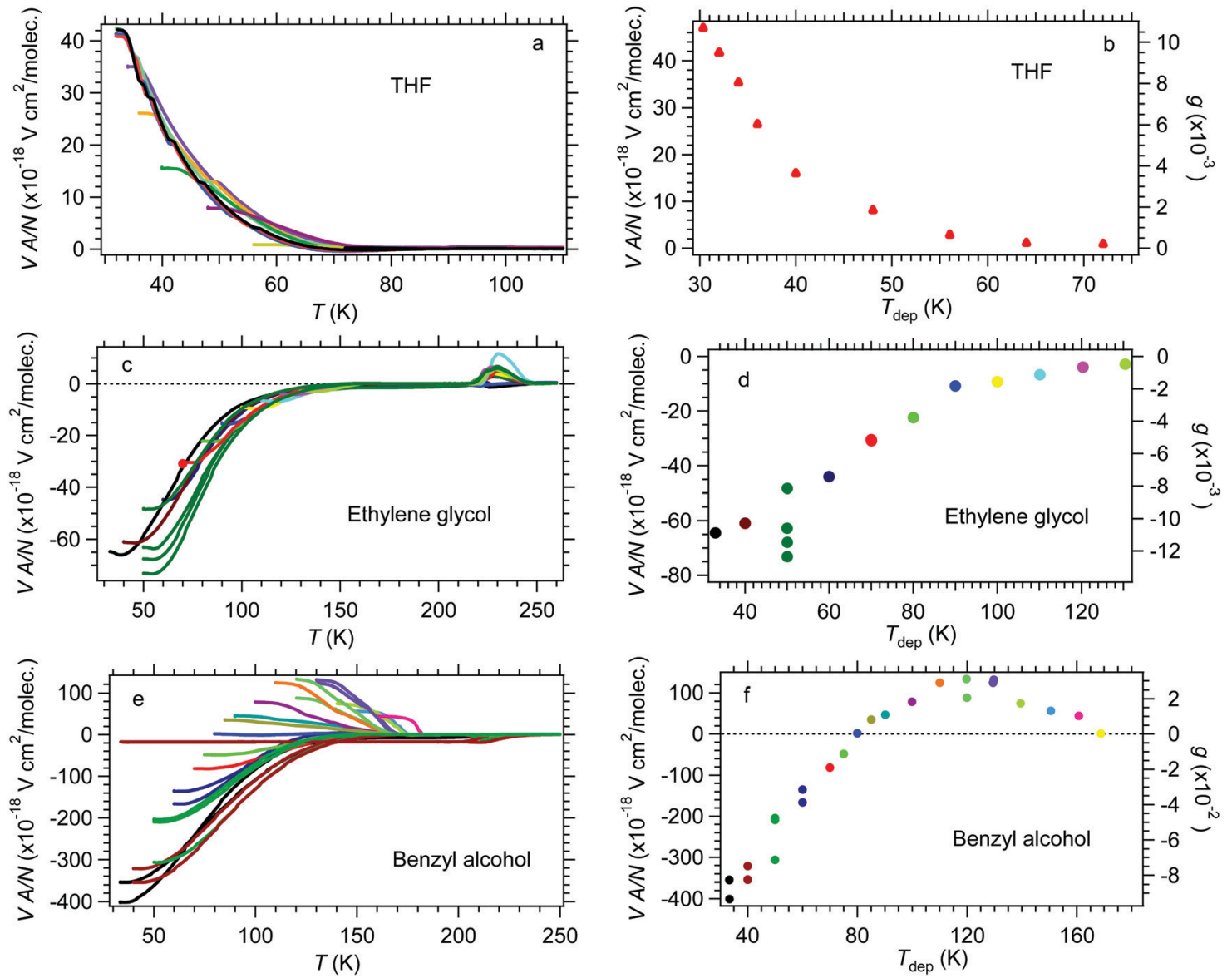

Fig. 1 ( $a, c$ and e) Voltage profiles during temperature ramps of films for named compound deposited at various temperatures have very similar shapes. Since film voltages scale with the deposited amount, data are displayed as measured voltages divided by surface number density of deposited molecules. (b, $\mathrm{d}$ and f) Initial scaled film voltage deposited at temperatures between $30.4 \mathrm{~K}$ and $72 \mathrm{~K}$ for THF, between $33 \mathrm{~K}$ and $130 \mathrm{~K}$ for benzyl alcohol, and between $33 \mathrm{~K}$ and $170 \mathrm{~K}$ for ethylene glycol and corresponding degree of polarization, calculated via eqn (1).

Ethylene glycol films showed negative film voltages at all deposition temperatures and rather small scaled voltages compared to other alcohols. The voltage profiles during heating ramps had similar shapes. A representative run is given in Fig. SA7 (ESI†), while several runs are collected in Fig. 1c. Initial film voltages for various deposition temperatures are shown in Fig. 2 d.

Benzyl alcohol films were deposited between 33 and $169 \mathrm{~K}$. A single run is shown in Fig. SA8 (ESI $\dagger$ ). The full range of depositions and temperature ramps is shown in Fig. 1e with the initial values of film voltages displayed in Fig. 1f. At the lower deposition temperatures film voltages are negative, dropping gradually and becoming 0 at $80 \mathrm{~K}$, but they grow again in the positive range reaching a maximum around $120 \mathrm{~K}$ and dropping again to 0 at higher temperatures. The heating profiles show two patterns. Up to about deposition temperature of $100 \mathrm{~K}$ these profiles are initially level, then they go smoothly to 0 . At temperatures above $140 \mathrm{~K}$ the film voltage is held constant for a wider temperature range followed by an abrupt voltage drop at a much higher ramp temperature. Films deposited between 100 and $140 \mathrm{~K}$ show an intermediate of these two patterns. The film grown at $80 \mathrm{~K}$ showed $0 \mathrm{~V}$, yet there were measurable fluctuations before film sublimation. Temperatures during ramp where film voltage has been reduced to half its initial value are shown in Fig. SC1, but they don't present a distinct pattern. The point at $80 \mathrm{~K}$ stands out because the corresponding initial film voltage was 0 . Overall, benzyl alcohol showed marked variations from all other alcohols studied so far.

Toluene films were deposited at temperatures between $32 \mathrm{~K}$ and $128 \mathrm{~K}$. A single run with simultaneous recording of the mass spectrometer signal is shown in Fig. SA9 (ESI $\dagger$ ). Most samples show a gradual elimination of a positive film voltage (see Fig. 3a, with initial voltages in Fig. 3g), except for deposition temperatures higher than $80 \mathrm{~K}$ where the voltage persists for a long temperature range followed by an abrupt drop. This pattern is also summarized in Fig. SC2 (ESI $\dagger$ ), where we report the temperature at which the initial voltage drops to half its value during the ramp and the slope with which it drops at that point. Unlike the other compounds presented so far, toluene shows a pronounced increase in initial voltage with increasing temperature up to $64 \mathrm{~K}$ and then shows a "normal" reduction of the voltage, but no change in voltage sign.

Methyl acetate films were deposited at temperatures between $33 \mathrm{~K}$ and $120 \mathrm{~K}$. A single run with the accompanying mass spectrometer signal is shown in Fig. SA10 (ESI $\dagger$ ). Fig. $3 \mathrm{~b}$ shows 

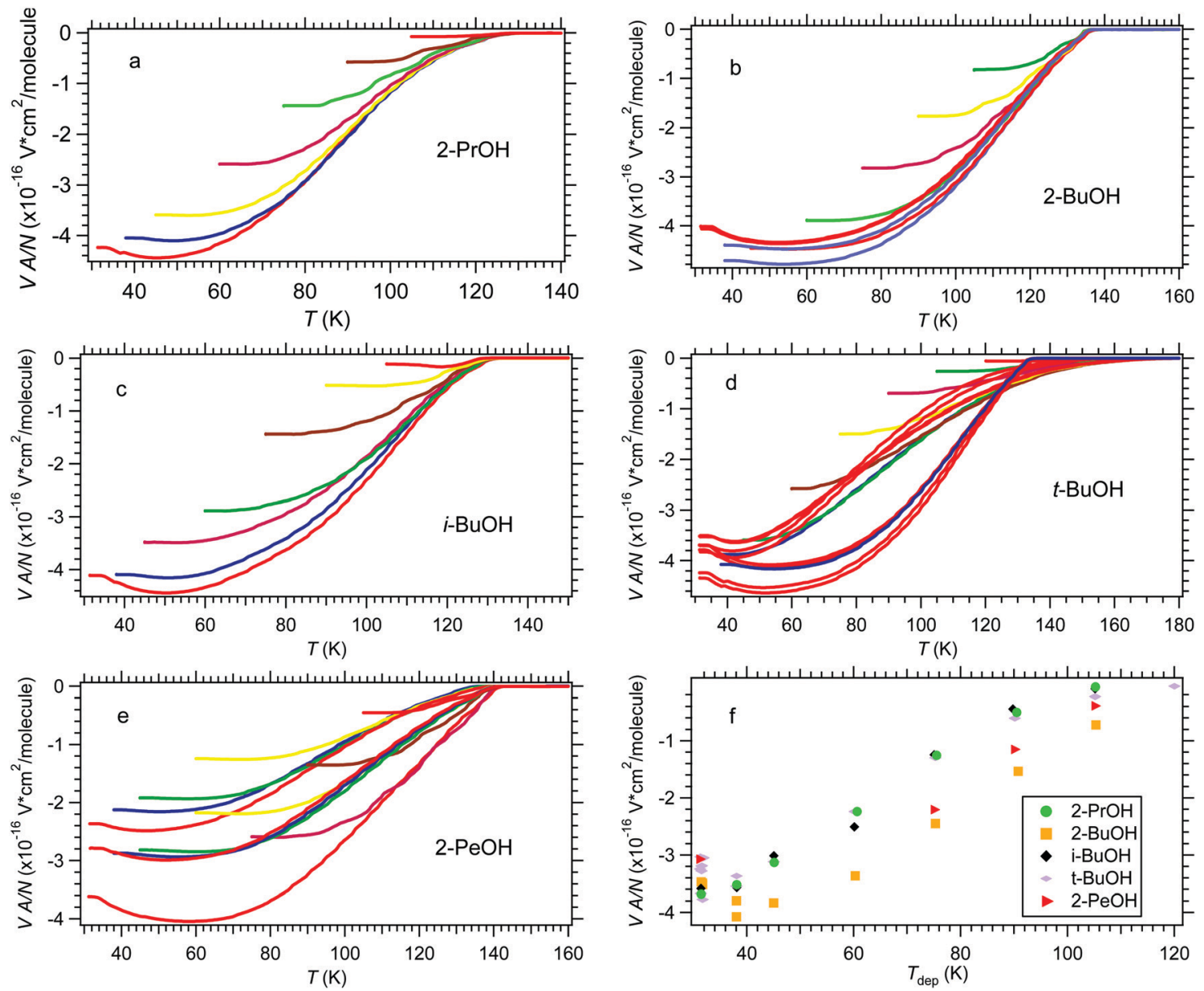

Fig. 2 (a-e) Voltage profiles during temperature ramps of films of named alcohol deposited at various temperatures have very similar shapes. Data are displayed as measured voltages divided by surface number density. (f) Initial scaled film voltage for each aliphatic alcohol deposited at temperatures between $31 \mathrm{~K}$ and $120 \mathrm{~K}$.

the scaled voltage profiles for all deposition temperatures. It is seen that the film voltage is positive in all cases and drops gradually during the ramp except for deposition at $100 \mathrm{~K}$ or higher where the drop comes late and is abrupt, very much like in the case of toluene. The initial film voltage variation with deposition temperature also looks a lot like that of toluene, with an increase up to around $60 \mathrm{~K}$ and a decrease thereafter (see Fig. SB3, ESI $\dagger$ ). The ramp temperature at which the film voltage is halved and the slope of the curve at that point show interesting variation with deposition temperature; these features are discussed below along with corresponding data from other acetate films.

Ethyl acetate films were deposited at temperatures between $33 \mathrm{~K}$ and $120 \mathrm{~K}$. A single run with the mass spectrometer signal is shown in Fig. SA11 (ESI†). Complete ramps of scaled film voltage are shown in Fig. 3c. Most of them exhibit gradual voltage drop, except for depositions at $100 \mathrm{~K}$ or higher. Initial film voltage rises with temperature up to $90 \mathrm{~K}$, then drops faster (see Fig. SB4, ESI†). Half drop temperatures show a regular pattern, but slopes at those points have a steep drop for deposition temperatures $90 \mathrm{~K}$ or higher (see Fig. SC4a, ESI $\dagger$ ). Overall, the situation with ethyl acetate films is very similar with that of methyl acetate films.
Propyl acetate was deposited at temperatures between $33 \mathrm{~K}$ and $120 \mathrm{~K}$. A single run with the mass spectrometer signal is shown in Fig. SA12 (ESI $\dagger$ ). Curves of scaled voltages for all the temperatures can be found in Fig. 3d. As seen in Fig. SB5 (ESI $\dagger$ ), initial voltage rises with deposition temperature up to $90 \mathrm{~K}$ and then drops fast. During the ramps the voltage drops gradually for depositions up to $90 \mathrm{~K}$, and later but more abruptly for higher deposition temperatures. The behaviour of propyl acetate is very similar to that of ethyl acetate with a slight shift of turning points to somewhat higher temperatures.

Butyl acetate films were formed at temperatures between $33 \mathrm{~K}$ and $130 \mathrm{~K}$. A single run showing the evolution of the film voltage during the temperature ramp and the mass spectrometer signal monitoring the gas phase is given in Fig. SA13 (ESI $\dagger$ ). Scaled film voltages for all deposition temperatures are found in Fig. 3e. Initial scaled voltages, summarized in Fig. SB6 (ESI $\dagger$ ), show always positive, rising values for deposition temperatures up to $90 \mathrm{~K}$ and a rapid drop beyond that temperature. During the ramps the voltage drops monotonically at a rate which accelerates as the deposition temperature rises (see Fig. SC4, ESI †).

Pentyl (or amyl) acetate films were grown at temperatures between $33 \mathrm{~K}$ and $130 \mathrm{~K}$. A single run with film voltage variation 

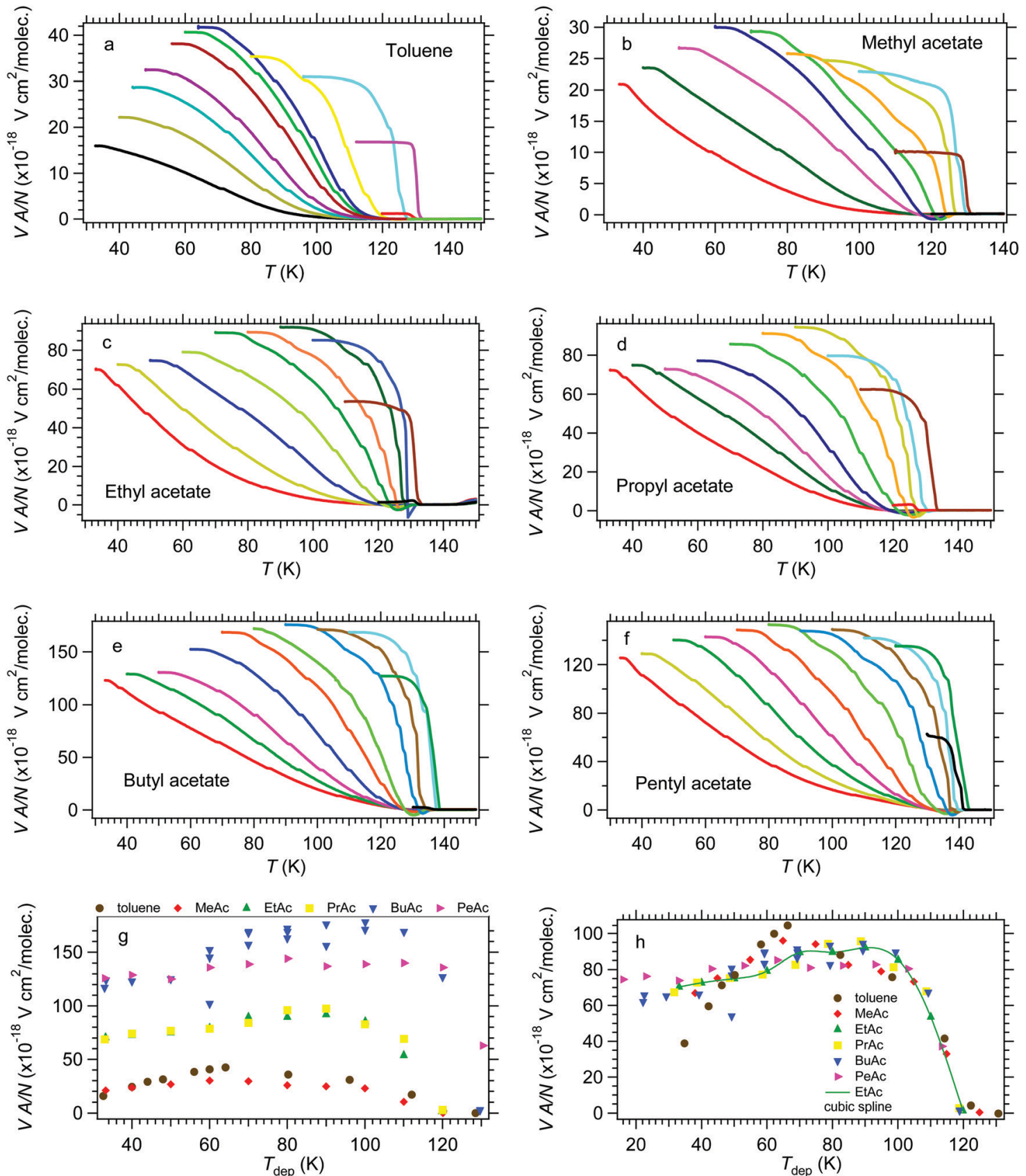

Fig. 3 (a-f) Scaled voltage profile for films of named compounds deposited at the lowest curve of each curve and ramped at $0.25 \mathrm{~K} \mathrm{~s}{ }^{-1}$ past the desorption temperature of each compound. (g) Initial scaled voltage for alkyl acetate and toluene films as a function of deposition temperature. (h) Data shown in panel $(\mathrm{g})$ shifted and scaled separately for each compound to match the profile of ethyl acetate data; fitting parameters used are shown in Table 2.

and mass spectrometer signal is given in Fig. SA14 (ESI $\dagger$ ). Complete scaled film voltage profiles during ramps for all deposition temperatures are shown in Fig. 3f. Initial voltages are summarized in Fig. SB7 (ESI $\dagger$ ). All films show positive initial voltages, rising with deposition temperature up to about $90 \mathrm{~K}$, beyond which temperature they drop off quickly. During the ramps, the voltage drops monotonically, slowly at low deposition temperature, much faster at higher temperatures. In all, the picture for pentyl acetate is very similar to that of butyl acetate.
Propanone (or acetone) was deposited at substrate temperatures ranging between $31 \mathrm{~K}$ and $120 \mathrm{~K}$. A typical example of a single run is shown in Fig. 1 . The acetone film exhibits a positive voltage of $3.4 \times 10^{-18} \mathrm{~V} \mathrm{~cm}^{2}$ per molecule for deposition at $30.8 \mathrm{~K}$. This value quickly drops to nearly 0 once the temperature ramp is started. Acetone is removed from the platinum foil above $150 \mathrm{~K}$, as seen with the mass spectrometer.

Several voltage measurements for acetone, scaled with the surface number density of the deposited molecules, are shown in Fig. 4a. Based on Fig. SA13 (ESI $\dagger$ ) and the trend seen for 

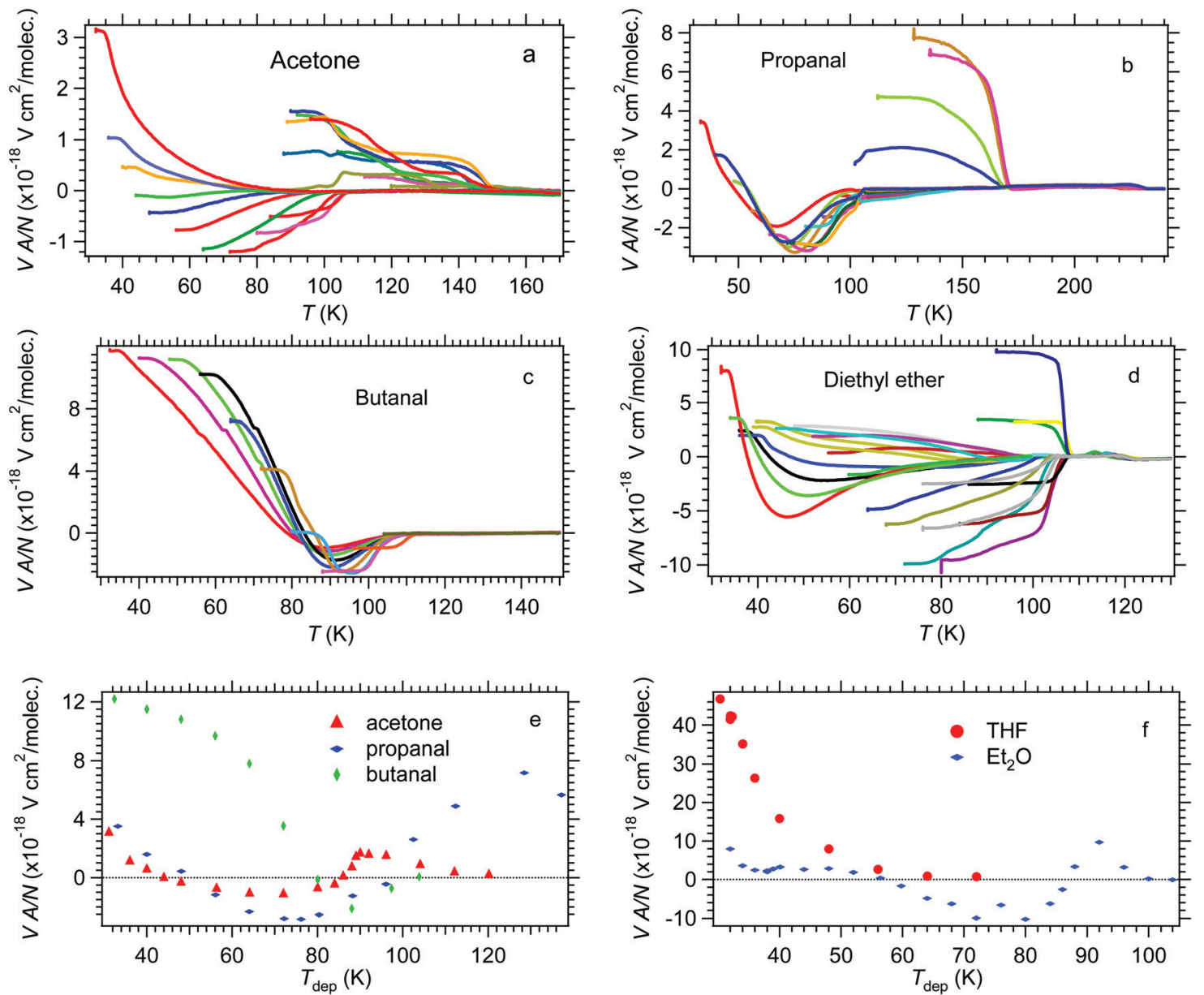

Fig. 4 (a-d) Deposition of named compound at different substrate temperatures leads to vastly different behaviour of the film voltage, both in terms of the sign and the magnitude of the initial voltage, as well as its variation during the subsequent temperature ramp. Lower panels: Initial scaled voltage for (e) acetone, aldehyde, and (f) ether films as a function of deposition temperature.

depositions up to about $40 \mathrm{~K}$, one would expect complete lack of film polarization above that temperature. Instead, films deposited at temperatures between $44 \mathrm{~K}$ and $84 \mathrm{~K}$ are polarized in the opposite direction, having negative voltages on the vacuum side. For deposition temperatures between $86 \mathrm{~K}$ and $120 \mathrm{~K}$ the polarization switches sign once more. In the latter range, the voltage persists during the ramp before disappearing. The most curious case is that of $86 \mathrm{~K}$ where the film voltage appears to be nearly 0 at first, but rises substantially during the temperature ramp. Initial film voltages are summarized in Fig. 4f and Fig. SB8 (ESI $\dagger$ ). Initial scaled voltage values are shown in Fig. SB6 as a function of deposition temperature. The same data can be interpreted as signed degree of polarization, $g$, based on eqn (1). The highest $g$ value of about $0.07 \%$ was observed at the lowest temperature achieved.

Propanal films were deposited at temperatures ranging between $33 \mathrm{~K}$ and $128 \mathrm{~K}$. The temperature ramp of the voltage of a sample formed at $33.2 \mathrm{~K}$, shown in Fig. SA16 (ESI $\dagger$ ) along with the desorption signal, exhibits a novel behaviour compared to all other compounds presented so far. The film bears positive voltage which turns negative during the temperature ramp. At higher deposition temperatures the initial voltage becomes less positive and eventually negative, while in each ramp the voltage drops further before fading away (see Fig. 4b and Fig. SB9a, ESI $\dagger$ ). Above $80 \mathrm{~K}$ the initial voltage turns less negative and it appears at $100 \mathrm{~K}$ it would disappear altogether. Instead, at higher deposition temperatures, the films exhibit positive voltages that persist during the ramps up to the sublimation of the film. For the films that show decrease in voltage before decaying to zero, we record the temperature of the minimum in Fig. SB9b (ESI $\dagger$ ). In all, propanal is strongly reminiscent of acetone.

Butanal films grown at temperatures between $32.4 \mathrm{~K}$ and $80 \mathrm{~K}$ have positive voltages that drop a little into the negative range during ramps (see Fig. SB17 (ESI $\dagger$ ) and Fig. 4c). For deposition temperatures between $88 \mathrm{~K}$ and $104 \mathrm{~K}$, film voltages are negative that gradually decay during the ramp ( $c f$. Fig. SB10a, ESI $\dagger$ ). Minimum voltage reached during ramp as well as the temperature at the minimum are shown in Fig. SB10b (ESI†). On the whole, butanal seems as a simplified version of propanal.

Fig. 4e shows a direct comparison of the voltage profiles for all three carbonyl bearing compounds. All three show negative film voltages at low deposition temperatures and negative higher ones and again positive voltages at even higher temperatures (at least the two three-carbon molecules). Butanal is the larger 
analogue of propanal and its films show higher voltages than propanal.

Diethyl ether films deposited at temperatures between $32 \mathrm{~K}$ and $56 \mathrm{~K}$ exhibit positive voltages (see Fig. SA18 (ESI $\dagger$ ) and Fig. 4d). Films formed below $38 \mathrm{~K}$ show a remarkable switch in the voltage sign. Films deposited above $56 \mathrm{~K}$ and below $88 \mathrm{~K}$ have a negative voltage which decays monotonically as the temperature is raised. For deposition temperatures between $88 \mathrm{~K}$ and $104 \mathrm{~K}$ films have positive voltages again which remain quite stable even at $100 \mathrm{~K}$. Diethyl ether films sublimate at temperatures higher than $140 \mathrm{~K}$, as seen in Fig. SA18 (ESI $\dagger$ ).

\section{Discussion}

We now take a look at trends and differences among the compounds studied. There is information to be gained from the film voltages at the deposition temperature, but we should be able to extract additional information from the fluctuations of the voltage during the temperature ramps.

By comparing all the scaled initial film voltages shown in Fig. 1-4, first we notice that these are not in the same order of magnitude. Monohydroxy alcohols, both aliphatic and aromatic, seem to present the highest voltages. Next in size come voltages from toluene, ester, ethylene glycol, and ether films. Carbonyl bearing compounds show the lowest film voltages. This observation fits with the general observation that dipole moment is required if a molecule is to form a polarized film, but voltage size does not correlate with dipole moment size, rather the opposite. Ethylene glycol sits at an interesting intersection; it is an alcohol and alcohols give rise to large voltages. At the same time, bearing two adjacent hydroxyl groups, it has a large dipole moment. The opposite trends place it in the middle of the range.

A rather solid conclusion is that among compounds of the same homologous series, increase in carbon chain length results in increased film voltage. This is clear among the alkyl acetates and has been reported for the normal monohydroxy alcohols. Note that the dipole moment does not vary substantially within a homologous series. Comparisons among the alcohols studied here are not so clear-cut because despite their differences in mass, they also differ in shape with branched carbon chains. At first glance one could argue that butanal has larger film voltage than propanal, but this statement can be made only for the lower range of deposition temperatures. At higher temperatures they follow very different trends making a comparison impossible.

Similarities in the initial film voltage vs. deposition temperature profiles among acetates begged the direct comparison shown in Fig. $3 \mathrm{~g}$. Toluene which seemed to exhibit the same profile is also place in the same figure. Common features among these data sets can be enhanced by trying to mold them into a common shape. This was achieved by means of a least squares fitting procedure. Data that were scaled vertically and shifted in temperature to match the shape of ethyl acetate data that were used as a template. Fig. $3 \mathrm{~h}$ confirms that all acetate profiles share a common shape. On the other hand, although toluene shares some of their crude features, it has a slightly
Table 2 Initial acetates voltage fit parameters based on EtAc

\begin{tabular}{lllll}
\hline Compound & scale & $\sigma$ & $\Delta T(\mathrm{~K})$ & $\sigma(\mathrm{K})$ \\
\hline Toluene & 2.5 & $(0.4)$ & -2 & $(7)$ \\
MeAc & 3.2 & $(0.7)$ & -5 & $(8)$ \\
EtAc & 1 & $(0)$ & 0 & $(0)$ \\
PrAc & 0.98 & $(0.07)$ & 1.4 & $(2.2)$ \\
BuAc & 0.53 & $(0.013)$ & 10.8 & $(1.1)$ \\
PeAc & 0.59 & $(0.02)$ & 17.0 & $(1.6)$
\end{tabular}

different pattern; its voltage starts very low and rises faster with temperature than acetate film voltages. Fit parameters and associated uncertainties are shown in Table 2.

In the acetone data the dramatic pattern change above $84 \mathrm{~K}$ (Fig. 4a) is expected to correlate with a significant change in film structure. This is not connected to the glass transition which is expect at a temperature almost $100 \mathrm{~K}$ higher.

The change in pattern for heating profiles of diethyl ether films above $88 \mathrm{~K}$ (see Fig. 4d) must be associated with changes in the film structure. In this case the glass transition temperature is reported very close to that temperature, namely $93 \mathrm{~K}$. Despite the enhanced mobility above the glass transition temperature, ether films are grown with non-zero polarization. All depositions above $88 \mathrm{~K}$ show a remarkable voltage stability up to $108 \mathrm{~K}$.

Fig. If displays the profiles of the two ether molecules studied in this work, namely, diethyl ether and tetrahydrofuran. Their spontaneous polarization shows a very different behaviour. These molecules have about the same mass, but THF is a rigid ring, whereas the other is a rather labile 5-membered chain. In contrast with other comparable molecules, here the molecule with the higher dipole moment exhibits higher film voltages. The low voltages and sign fluctuations seen in diethyl ether may be attributed to its flexible form.

We mention in passing that we attempted experiments with mixtures of toluene with helium, which was not expected to interact with the substrate due to its extremely low boiling point. They aimed to identify any effect under conditions deviating from vacuum. Scaling used in Fig. SB2 (ESI $\dagger$ ) relied on the net amount of toluene deposited, but results were not conclusive.

All compounds fail to produce film voltages near the sublimation temperature. For some compounds, such as THF or the aliphatic alcohols, deposition voltages and heating profiles die out at the same temperatures and all voltages maintain their sign. For others, though, by looking at the heating profiles for low temperature deposition one would never guess that they could show non-zero film voltages for deposition at certain high temperatures and, even more, preserve them during heating. For example, a benzyl alcohol film deposited at $40 \mathrm{~K}$ reaches $0 \mathrm{~V}$ around $140 \mathrm{~K}$; nonetheless, films deposited at between 140 and $160 \mathrm{~K}$ show measurable voltages. As already mentioned, the most curious case is that of acetone deposited at $86 \mathrm{~K}$ with $0 \mathrm{~V}$ at first that grows during heating.

One of the most puzzling features is what makes the measured electrical polarization change sign as the film temperature is raised. It is common knowledge that any change is driven by energy minimization. Just as crystallization of amorphous 
solids occurs when energy barriers are surmounted and the new structure is at a lower energy, we need to look for mechanisms that rearrange the molecules, move atoms or simply shuffle around electrons by some small extent enough though to tip a balance. We need to remember that the degree of polarization is of the order of $10^{-3}$ to $10^{-2}$, which means that a very small projection of the dipole moment vector is perpendicular to the substrate plane. A slight shift of dipole moment vector orientation can cause the measured voltage to change sign. Elimination of film voltage indicates randomization of dipole moment vector orientation, whereas polarization sign change indicates maintenance of vector correlation with a concurrent small turn with respect to the surface normal. When this happens, film voltage is a small effect in the total energy balance, but it is our simple means of detecting orientation changes.

Acetate film voltages show an interesting pattern as they drop towards zero during sample heating. The temperature at half point of the drop is shown in Fig. SC3 (ESI $\dagger$ ), whereas the slope of the voltage at that point in presented in Fig. SC4a $(\mathrm{ESI} \dagger)$ for all acetate and toluene films. Slope variation with temperature for each film has the same pattern, but different size. We attempted scaling and shifting of these points in order to maximize overlap. We implemented a least squares fitting procedure using as a template an interpolated curve for the ethyl acetate data. The results are shown in Fig. SC4b and in Table SC1 (ESI $\dagger$ ). The last column is the deposition temperature giving rise to the steepest slope as calculated from the shift and the corresponding point of the template curve. Following the same procedure, but using as template the butyl acetate data yielded similar results also shown in the right half side of Table SC1 and drawn in Fig. SC4c (ESI $\dagger$ ).

Going one step further, we looked at the temperature derivative of the voltage profiles during sample heating. Any drastic change in structure or function (e.g. glass transition) is expected to cause an anomalous fluctuation in the voltage. ${ }^{30}$ Results for most of our data are shown in Fig. SD1-SD3 (ESI $\dagger$ ). They appear to be noisy and no numerical smoothing has been applied. The same is true for any of the raw data.

Once more THF is the simplest case, with no outstanding features. Benzyl alcohol doesn't hold many surprises except for a strong peak at $179 \mathrm{~K}$ only for films deposited at $160 \mathrm{~K}$. If this peak denotes the glass transition, it has come about $10 \mathrm{~K}$ later that the value reported in the literature. A similar statement can be made for toluene. Deposition at 96 and $112 \mathrm{~K}$, i.e., just below $T_{\mathrm{g}}$ at $117 \mathrm{~K}$, gives rise to a derivative peak at 125 and $130 \mathrm{~K}$ respectively, whereas deposition at $120 \mathrm{~K}$ gives very small voltage and a small derivative peak at $130 \mathrm{~K}$. Delayed appearance of glass transition is in line with other experiments looking at thermodynamic stability of vapour-deposited films.

Sets of two peaks in the derivate plots appeared in films deposited at higher temperatures, possibly indicating a dual nature of films grown. Derivative plots of films other than esters were flat in general except for high temperature film depositions (e.g. benzyl alcohol and diethyl ether). It would be interesting if fictive temperatures ${ }^{79,80}$ could be determined from these plots, but there is no obvious way this could be done.
One possibility could be considered in the cases of voltage profiles that go to zero at low temperatures, which point could be taken as the fictive temperature for these films. In these cases the argument would be that when the voltage dies out, the sample has turned isotropic, i.e., liquid-like.

\section{Conclusions}

Spontaneous polarization generated in cryo-deposited films was studied for a wide range of small organic molecules at deposition temperatures ranging from $30 \mathrm{~K}$ to $130 \mathrm{~K}$. Initial voltages varied is sign and magnitude depending on the homologous series, molecule size, and deposition temperature. Raising the substrate temperature after deposition causes varying voltage fluctuations and eventually leads to irreversible elimination of the voltage prior to film evaporation.

Quantitative comparisons of initial scaled film voltages reveal interesting trends and differences among different compounds. Toluene and the alkyl acetates share many similarities in overall behaviour. Over the deposition temperature range studied their films show positive initial voltages (like $\mathrm{SO}_{2}{ }^{1}$ $\mathrm{N}_{2} \mathrm{O},{ }^{9}$ methyl formate ${ }^{13}$ ) which rise and fall with increasing deposition temperature. Tetrahydrofuran follows a simpler trend of positive voltages decreasing with rising temperatures, although that could simply be a consequence of the accessible temperatures on the low side. The same could be said for the monohydroxy aliphatic alcohols studied which form negative films (like water ${ }^{9}$ ). The heavier members of the series show a decrease in the absolute value of initial voltage at the very low temperatures. It is possible that the lighter members could show the same trend at temperatures below $30 \mathrm{~K}$. Comparison among $n$-alkyl acetates shows the same trend as that observed for primary alcohols, ${ }^{29}$ i.e., the larger the molecule, the larger the film voltage (see Fig. 13). Variations both in magnitude and sign have been reported for $\mathrm{CO}, \mathrm{NO}$, and acetone from the first systematic study of spontaneous polarization. ${ }^{1}$

Estimated degrees of polarization, $g$, are based on assumptions regarding the dipole moment and the relative dielectric constant at infinite frequency. The values determined indicate that only a small fraction of deposited molecules align their dipole moment vector perpendicular to the substrate surface. A general trend is that $g$ is lower for molecules with high dipole moment. This might be due to stronger intermolecular interactions among molecules with high dipole moment; a striking example is that of ethylene glycol which can form hydrogen bonds on both ends. Weaker intermolecular forces might allow molecules to move more freely when they land on the cold substrate, before they become immobilized by subsequent deposition of other layers.

Changes in film voltage evolution with ramp temperature indicate changes in the structure of deposited films. Delayed reduction of the voltage indicates higher stability of the film, because energy barriers to rearrangement are higher. What is harder to explain is the increase of voltage during a ramp or, more spectacular, when the voltage changes sign, as seen in propanal, butanal and diethyl ether films. Interpretation of these 
features will likely require complementary measurements, but more importantly computer simulations.

\section{Conflicts of interest}

There are no conflicts to declare.

\section{Acknowledgements}

We thank Dr J. P. Cowin, Pacific Northwest National Laboratory for making several pieces of equipment available to us for use. A. A. T. thanks the Empirikion Foundation and the National and Kapodistrian University of Athens, Special Account for Research Grants for funding provided. I. K. G. acknowledges the scholarship provided by the Greek State Scholarship Foundation with funding from the European Social Fund. Finally, we thank the two reviewers for prompt, detailed, insightful and helpful comments.

\section{References}

1 K. Kutzner, Thin Solid Films, 1971, 14, 49.

2 L. Onsager, D. L. Staebler and S. Mascarenhas, J. Chem. Phys., 1978, 68, 3823.

3 B. Sujak and J. Chrzanowski, Thin Solid Films, 1980, 71, 47. 4 J. Chrzanowski and B. Sujak, Thin Solid Films, 1981, 79, 101. 5 J. Chrzanowski and B. Sujak, Thin Solid Films, 1983, 101, 123. 6 J. Chrzanowski and B. Sujak, Thin Solid Films, 1983, 103, 417. 7 J. Chrzanowski and B. Sujak, Cryogenics, 1983, 23, 91.

8 W. J. Sobolewski, Phase Transitions, 1997, 62, 95.

9 M. J. Iedema, M. J. Dresser, D. L. Doering, J. B. Rowland, W. P. Hess, A. A. Tsekouras and J. P. Cowin, J. Phys. Chem. B, 1998, 102, 9203.

10 R. Balog, P. Cicman, N. C. Jones and D. Field, Phys. Rev. Lett., 2009, 102, 073003.

11 R. Balog, P. Cicman, D. Field, L. Feketeov, K. Hoydalsvik, N. C. Jones, T. A. Field and J.-P. Ziesel, J. Phys. Chem. A, 2011, 115, 6820.

12 O. Plekan, A. Cassidy, R. Balog, N. C. Jones and D. Field, Phys. Chem. Chem. Phys., 2011, 13, 21035.

13 O. Plekan, A. Cassidy, R. Balog, N. C. Jones and D. Field, Phys. Chem. Chem. Phys., 2012, 14, 9972.

14 A. Cassidy, O. Plekan, R. Balog, N. C. Jones and D. Field, Phys. Chem. Chem. Phys., 2013, 15, 108.

15 D. Field, O. Plekan, A. Cassidy, R. Balog, N. C. Jones and J. Dunger, Int. Rev. Phys. Chem., 2013, 32, 345.

16 A. Cassidy, O. Plekan, R. Balog, J. Dunger and D. Field, J. Phys. Chem. A, 2014, 119, 6615.

17 A. Cassidy, O. Plekan, J. Dunger, R. Balog, N. C. Jones, J. Lasne, A. Rosu-Finsen, M. R. S. McCoustra and D. Field, Phys. Chem. Chem. Phys., 2014, 16, 23843.

18 J. Lasne, A. Rosu-Finsen, A. Cassidy, M. R. S. McCoustra and D. Field, Phys. Chem. Chem. Phys., 2015, 17, 20971.

19 J. Lasne, A. Rosu-Finsen, A. Cassidy, M. R. S. McCoustra and D. Field, Phys. Chem. Chem. Phys., 2015, 17, 30177.
20 A. Rosu-Finsen, J. Lasne, A. Cassidy, M. R. S. McCoustra and D. Field, Phys. Chem. Chem. Phys., 2016, 18, 5159.

21 A. Cassidy, Mads R. V. Jorgensen, A. Rosu-Finsen, J. Lasne, J. H. Jorgensen, A. Glavic, V. Lauter, Bo B. Iversen, M. R. S. McCoustra and D. Field, J. Phys. Chem. C, 2016, 120, 14130.

22 A. Rosu-Finsen, J. Lasne, A. Cassidy, M. R. S. McCoustra and D. Field, Astrophys. J., 2016, 832, 1.

23 I. K. Gavra, A. N. Pilidi and A. A. Tsekouras, J. Chem. Phys, 2017, 146, 104701.

24 O. Plekan, A. Rosu-Finsen, A. M. Cassidy, J. Lasne, M. R. S. McCoustra and D. Field, Eur. Phys. J. D, 2017, 71, 162.

25 M. Roman, S. Taj, M. Gutowski, M. R. S. McCoustra, A. C. Dunn, Z. G. Keolopile, A. Rosu-Finsen, A. M. Cassidy and D. Field, Phys. Chem. Chem. Phys., 2018, 20, 5112.

26 M. Roman, A. Dunn, S. Taj, Z. G. Keolopile, A. Rosu-Finsen, M. Gutowski, M. R. S. McCoustra, A. M. Cassidy and D. Field, Phys. Chem. Chem. Phys., 2018, 20, 29038.

27 A. Cassidy, R. L. James, A. Dawes, J. Lasne and D. Field, Phys. Chem. Chem. Phys., 2019, 21, 1190.

28 D. Field, Phys. Chem. Chem. Phys., 2019, 21, 26606.

29 A. N. Pilidi, I. K. Gavra and A. A. Tsekouras, J. Phys. Chem. B, 2019, 123, 8505.

30 R. Sagi, M. Akerman, S. Ramakrishnan and M. Asscher, J. Chem. Phys., 2020, 153, 124707.

31 R. Sagi, M. Akerman, S. Ramakrishnan and M. Asscher, J. Chem. Phys., 2020, 153, 144702.

32 E. Ito, Y. Washizu, N. Hayashi, H. Ishii, N. Matsuie, K. Tsuboi, Y. Ouchi, Y. Harima, K. Yamashita and K. Seki, J. Appl. Phys., 2002, 92, 7306.

33 K. Sugi, H. Ishii, Y. Kimura, M. Niwano, E. Ito, Y. Washizu, N. Hayashi, Y. Ouchi and K. Seki, Thin Solid Films, 2004, 464-465, 412.

34 K. Yoshizaki, T. Manaka and M. Iwamoto, J. Appl. Phys., 2005, 97, 023703.

35 N. Kajimoto, T. Manaka and M. Iwamoto, J. Appl. Phys., 2006, 100, 053707.

36 E. Ito, T. Isoshima, K. Ozasa and M. Hara, Mol. Cryst. Liq. Cryst., 2007, 462, 111.

37 T. Isoshima, H. Ito, E. Ito, Y. Okabayashi and M. Hara, Mol. Cryst. Liq. Cryst., 2009, 505, 59.

38 Y. Okabayashi, E. Ito, T. Isoshima, H. Ito and M. Hara, Thin Solid Films, 2009, 518, 839.

39 A. Lukyanov, C. Lennartz and D. Andrienko, Phys. Status Solidi A, 2009, 206, 2737.

40 Y. Noguchi, Y. Miyazaki, Y. Tanaka, N. Sato, Y. Nakayama, T. D. Schmidt, W. Brütting and H. Ishii, J. Appl. Phys., 2012, 111, 114508.

41 Y. Okabayashi, E. Ito, T. Isoshima and M. Hara, Appl. Phys. Express, 2012, 5, 055601.

42 T. Isoshima, Y. Okabayashi, E. Ito, M. Hara, W. W. Chin and J. W. Han, Org. Electron., 2013, 14, 1988.

43 M. Mladenovic and N. Vukmirovic, J. Phys. Chem. C, 2016, 120, 18895.

44 Y. Esaki, T. Komino, T. Matsushima and C. Adachi, J. Phys. Chem. Lett., 2017, 8, 5891. 
45 K. Osada, K. Goushi, H. Kaji, C. Adachi, H. Ishii and Y. Noguchi, Org. Electron., 2018, 58, 313.

46 Y. Noguchi, W. Brutting and H. Ishii, Jpn. J. Appl. Phys., 2019, 58, SF0801.

47 K. Ishii and H. Nakayama, Phys. Chem. Chem. Phys., 2014, 16, 12073.

48 K. L. Kearns, S. F. Swallen, M. D. Ediger, T. Wu and L. Yu, J. Chem. Phys., 2007, 127, 154702.

49 M. D. Ediger and P. Harrowell, J. Chem. Phys., 2012, 137, 080901.

50 S. F. Swallen, K. L. Kearns, M. K. Mapes, Y. S. Kim, R. J. McMahon, M. D. Ediger, T. Wu, L. Yu and S. Satija, Science, 2007, 315, 353.

51 K. L. Kearns, S. F. Swallen, M. D. Ediger, T. Wu, Y. Sun and L. Yu, J. Phys. Chem. B, 2008, 112, 4934.

52 L. Zhu, C. W. Brian, S. F. Swallen, P. T. Straus, M. D. Ediger and L. Yu, Phys. Rev. Lett., 2011, 106, 256103.

53 K. J. Dawson, L. Zhu, L. Yu and M. D. Ediger, J. Phys. Chem. $B, 2011,115,455$.

54 S. S. Dalal and M. D. Ediger, J. Phys. Chem. Lett., 2012, 3, 1229.

55 I. Lyubimov, M. D. Ediger and J. J. de Pablo, J. Chem. Phys., 2013, 139, 144505.

56 S. S. Dalal, D. M. Walters, I. Lyubimov, J. J. de Pablo and M. D. Ediger, Proc. Natl. Acad. Sci. U. S. A., 2015, 112, 4227.

57 M. Tylinski, Y. Z. Chua, M. S. Beasley, C. Schick and M. D. Ediger, J. Chem. Phys., 2016, 145, 174506.

58 M. D. Ediger, J. Chem. Phys., 2017, 147, 210901.

59 D. M. Walters, L. Antony, J. J. de Pablo and M. D. Ediger, J. Phys. Chem. Lett., 2017, 8, 3380.

60 Y. Chen, Z. Chen, M. Tylinski, M. D. Ediger and L. Yu, J. Chem. Phys., 2019, 150, 024502.

61 K. Bagchi, N. E. Jackson, A. Gujral, C. Huang, M. F. Toney, L. Yu, J. J. de Pablo and M. D. Ediger, J. Phys. Chem. Lett., 2019, 10, 164.
62 M. D. Ediger, J. de Pablo and L. Yu, Acc. Chem. Res., 2019, 52, 407.

63 B. Riechers, A. Guiseppi-Elie, M. D. Ediger and R. Richert, J. Chem. Phys., 2019, 150, 214502.

64 C. Bishop, A. Gujral, M. F. Toney, L. Yu and M. D. Ediger, J. Phys. Chem. Lett., 2019, 10, 3536.

65 B. J. Kasting, M. S. Beasley, A. Guiseppi-Elie, R. Richert and M. D. Ediger, J. Chem. Phys., 2019, 151, 144502.

66 B. Riechers, A. Guiseppi-Elie, M. D. Ediger and R. Richert, J. Chem. Phys., 2019, 151, 174503.

67 C. Bishop, Y. Li, M. F. Toney, L. Yu and M. D. Ediger, J. Phys. Chem. B, 2020, 124, 2505.

68 K. Bagchi and M. D. Ediger, J. Phys. Chem. Lett., 2020, 11, 6935.

69 M. S. Beasley, B. J. Kasting, M. E. Tracy, A. Guiseppi-Elie, R. Richert and M. D. Ediger, J. Chem. Phys., 2020, 153, 124511.

70 K. Bagchi, M. E. Fiori, C. Bishop, M. F. Toney and M. D. Ediger, J. Phys. Chem. B, 2021, 125, 461.

71 J. P. Gabriel, B. Riechers, E. Thoms, A. Guiseppi-Elie, M. D. Ediger and R. Richert, J. Chem. Phys., 2021, 154, 024502.

72 S. Singh and J. J. de Pablo, J. Chem. Phys., 2011, 134, 194903. 73 M. Jorge and L. Lue, J. Chem. Phys., 2019, 150, 084108.

74 CRC Handbook of Chemistry and Physics, 83rd edn, ed. D. R. Lide, CRC Press, Boca Raton, 2002.

75 A. V. Lesikar, J. Phys. Chem., 1976, 80, 1005.

76 S. S. N. Murthy and S. K. Nayak, J. Chem. Phys., 1993, 99, 5362. 77 O. Yamamuro, I. Tsukushi, A. Lindqvist, S. Takahara, M. Ishikawa and T. Matsuo, J. Phys. Chem. B, 1998, 102, 1605. 78 J. V. Koleske and J. A. Faucher, Polym. Eng. Sci., 1979, 19, 716. 79 J. Ràfols-Ribé, M. Gonzalez-Silveira, C. Rodríguez-Tinoco and J. Rodríguez-Viejo, Phys. Chem. Chem. Phys., 2017, 19, 11089.

80 C. Rodríguez-Tinoco, M. Gonzalez-Silveira, J. Ràfols-Ribé, A. F. Lopeandía and J. Rodríguez-Viejo, Phys. Chem. Chem. Phys., 2015, 17, 31195-31201. 